\title{
Functional MRI as an Objective Measure of Olfaction Deficit in Patients with Traumatic Anosmia
}

\author{
(D)W.-J. Moon, (DM. Park, (DM. Hwang, and DJ.K. Kim
}

\begin{abstract}
BACKGROUND AND PURPOSE: While posttraumatic anosmia is not uncommon, the olfactory function evaluation has strongly relied on subjective responses given by patients. We aimed to examine the utility of $\mathrm{fMRI}$ as an objective tool for diagnosing traumatic anosmia.
\end{abstract}

MATERIALS AND METHODS: Sixteen patients (11 men and 5 women; mean age, $42.2 \pm 10.4$ years) with clinically diagnosed traumatic anosmia and 19 healthy control subjects (11 men and 8 women; mean age, $29.3 \pm 8.5$ years) underwent fMRI during olfactory stimulation with citral (a pleasant odor) or $\beta$-mercaptoethanol (an unpleasant odor). All patients were subjected to a clinical olfactory functional assessment and nasal endoscopic exploration. Two-sample $t$ tests were conducted with age as a covariate to examine group differences in brain activation responses to olfactory stimulation (false discovery rate-corrected $P<.05$ ).

RESULTS: Compared with healthy control subjects, patients with traumatic anosmia had reduced activation in the bilateral primary and secondary olfactory cortices and the limbic system in response to $\beta$-mercaptoethanol stimulation, whereas reduced activation was observed only in the left frontal subgyral region in response to citral stimulation.

CONCLUSIONS: Brain activation was decreased in the bilateral primary and secondary olfactory cortices as well as the limbic system in response to olfactory stimulation in patients with traumatic anosmia compared with healthy control subjects. These preliminary results may shed light on the potential of FMRI for the diagnosis of traumatic anosmia.

ABBREVIATIONS: $\mathrm{BME}=\beta$-mercaptoethanol; $\mathrm{BOLD}=$ blood oxygen level-dependent; $\mathrm{CC}$-SIT $=$ Cross-Cultural Smell Identification Test; $\mathrm{HC}=$ healthy control; KVSS $=$ Korean Version of the Sniffin' Sticks; MNI = Montreal Neurological Institute

$\mathbf{T}$ raumatic anosmia is not uncommon, affecting an estimated $5 \%-14.5 \%$ of patients with head trauma. ${ }^{1}$ The main mechanism of olfactory dysfunction after closed head trauma is thought to involve damage to the olfactory nerve and associated nerve centers. ${ }^{2}$ Traumatic anosmia can significantly affect quality of life,

Received June 7, 2018; accepted after revision September 24.

From the Departments of Radiology (W.-J.M., M.P.) and Otorhinolaryngology-Head and Neck Surgery (J.K.K.), Konkuk University Medical Center, Konkuk University School of Medicine, Seoul, Korea; and GE Healthcare (M.H.), Seoul, Korea.

This research was supported by Basic Science Research Program through the National Research Foundation of Korea (NRF) funded by the Ministry of Education (NRF-2016RID1A1B01012705).

Paper previously presented as a scientific poster at: Annual Meeting of the Radiological Society of North America, November 27 to December 1, 2016; Chicago, Illinois.

Please address correspondence to Jin Kook Kim, MD, PhD, Department of Otorhinolaryngology-Head and Neck Surgery, Konkuk University Medical Center, Konkuk University School of Medicine, 120-1, Neungdong-ro, Hwayang-dong, Gwangjin-gu, Seoul 05030, Korea South; e-mail: entalk@kuh.ac.kr

- Indicates open access to non-subscribers at www.ajnr.org

三 Indicates article with supplemental on-line tables.

Indicates article with supplemental on-line photo.

http://dx.doi.org/10.3174/ajnr.A5873 reducing food appreciation and impairing an individual's ability to detect environmental hazards. ${ }^{1,3}$ Traumatic anosmia is most commonly diagnosed through a psychophysical olfactory function test that primarily relies on patient self-reporting using questionnaires and simple odorant sticks or solutions. This semi-objective psychophysical test thus requires patient cooperation and intact cognition. ${ }^{4}$ In addition to olfactory loss, odor memory may also affect test results, ${ }^{4}$ which limits the diagnostic accuracy in patients with impaired cognition. Furthermore, due to its selfreporting nature, the psychophysical test is also vulnerable to manipulation by malingering patients involved in compensation litigation. ${ }^{5}$ Several studies have evaluated systematic and objective tools for assessing olfactory function in patients; in particular, previous work has examined the diagnostic utility of single-photon emission CT and MR imaging. Previous MR imaging studies in patients diagnosed with traumatic anosmia based on the clinical olfactory test have found that $61 \%-88 \%$ of the study population had gross damage to the olfactory system, which differed according to the degree of trauma. ${ }^{6-8}$ Studies using SPECT have detected abnormalities that were previously undetectable on MR 
imaging in traumatic anosmia; however, patients with traumatic normosmia exhibited similar abnormalities, limiting the diagnostic utility of SPECT. ${ }^{9,10}$

Functional MR imaging is a method that noninvasively evaluates the working human brain by detecting changes in blood oxygen level-dependent (BOLD) signal arising from neuronal responses to recurrent stimulation. Odor-stimulated or olfactory fMRI was first introduced by Yousem et $\mathrm{al}^{11}$ and is now used for the study of olfactory deficits in neurodegenerative diseases, schizophrenia, and congenital hyposmia. ${ }^{12-16}$ However, the exact utility of olfactory fMRI for the evaluation of traumatic anosmia is unclear. The aim of this study was to compare brain activation between patients with traumatic anosmia and healthy control subjects during olfactory fMRI with 2 different olfactory stimuli (a pleasant odor and an unpleasant odor) to determine whether fMRI can be used to objectively measure olfactory function in a diagnostic capacity.

\section{MATERIALS AND METHODS \\ Participants}

This prospective study was approved by the ethics committee of Konkuk University Medical Center. Patients with traumatic anosmia were recruited from the otorhinolaryngology department from November 2012 to February 2015. We enrolled 16 patients with traumatic anosmia (11 men and 5 women; mean age, $42.2 \pm$ 10.4 years). The inclusion criteria for patients with traumatic anosmia were as follows: 1) recent head injury, 2) criteria for anosmia met on the basis of the Korean Version of the Sniffin' Sticks (KVSS) II test, and 3) age between 18 and 65 years. Patients did not have any previous olfactory impairment, a history of sinonasal disease, or current nasal symptoms. Additionally, patients completed psychophysical olfactory testing, including the Cross-Cultural Smell Identification Test (CC-SIT), the KVSS I, and the KVSS II, and underwent nasal endoscopy to preclude the possibility of obstructive olfactory loss. Each participant provided written informed consent before study participation.

We also recruited 19 healthy control (HC) subjects (11 men and 8 women; mean age, $29.3 \pm 8.5$ years) from the local community. The inclusion criteria for HC participants were as follows: 1) normal olfactory function, 2) no brain lesions or prior substantial head trauma, and 3) no history of psychiatric or neurologic disease. All study participants were right-handed.

\section{Clinical Olfactory Assessment}

An otorhinolaryngologist with 17 years of clinical experience administered an examination to all patients that included an endoscopic examination of the nasal cavity and clinical olfactory performance testing to ensure that patients met the criteria for anosmia. Clinical olfactory performance measures included the CC-SIT, the KVSS I, and the KVSS II. ${ }^{17}$ The KVSS test is a modified Sniffin' Sticks test optimized for Korean patients, including the use of odors familiar to Korean individuals. ${ }^{17,18}$ The KVSS I is a test for rapid screening, and the KVSS II is the Korean equivalent of the Sniffin' Sticks test. ${ }^{17,18}$ Clinical diagnosis of anosmia was based on the KVSS II score, in which total scores of 0-20 were classified as anosmia, scores of 20.25-27 were classified as hyposmia, and scores of 27.25-48 were classified as normosmia. ${ }^{17,18}$
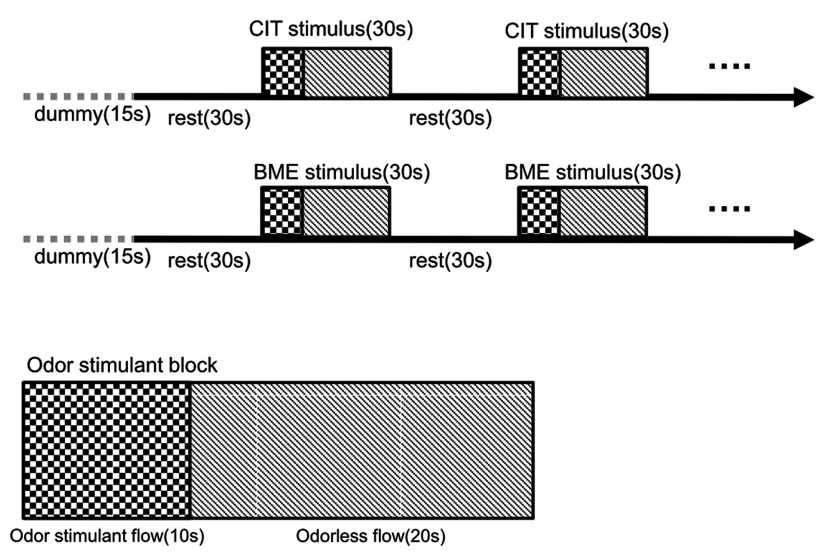

FIG 1. Task design of the citral (CIT) and $\beta$-mercaptoethanol (BME)stimulated olfactory tasks.

\section{MR Imaging Data Acquisition}

All participants underwent MR imaging with a 3T scanner (Signa HDxT; GE Healthcare, Milwaukee, Wisconsin) using an 8-channel head coil. High-spatial-resolution T1-weighted 3D anatomic images were obtained in the axial plane using a fast-spoiled gradient-recalled sequence (TR, $7.8 \mathrm{~ms}$; TE, $3.0 \mathrm{~ms}$; matrix, $256 \times$ 256; flip angle, $13^{\circ}$; number of sections, 134; FOV, $240 \times 240$ $\mathrm{mm}^{2}$; section thickness, $1.3 \mathrm{~mm}$ ).

The sequence for functional images was gradient recalled echo-planar imaging (TR, 3000 ms; TE, 35 ms; matrix, $64 \times 64$; flip angle, $90^{\circ}$; FOV, $240 \times 240 \mathrm{~mm}^{2}$; section thickness, $3.5 \mathrm{~mm}$ with no gap). A total of 100 brain volume sequences were collected during 5 minutes. Slices were aligned parallel to the anterior/posterior commissure line.

Functional images were subsequently obtained in a block design consisting of 5 odor exposure blocks ("on-period") and 5 normal breathing blocks ("off-period”). Odors were presented to participants via a custom-built olfactometer with continuous air flow (4 L/min) (Fig 1). During the 30 seconds on-period, odors were delivered to both nostrils for 10 seconds followed by $20 \mathrm{sec}-$ onds of odorless air with 50\% relative humidity at room temperature. However, it takes 3 seconds for the odor to travel through the tube from the odor container to the nasal piece; therefore, the on-period, in effect, consisted of the initial 3 seconds of odorless flow, 10 seconds of odor-stimulant flow, followed by 17 seconds of odorless flow. During the 30 seconds off-period, the subject also continuously received odorless air with 50\% relative humidity at room temperature. By infusing the odorants for only 10 of the 30 seconds in the on-period, we aimed to reduce the possibility of olfactory habituation.

In the first session, $0.2 \mathrm{~mL}$ of $10 \mathrm{mmol} / \mathrm{L}$ citral (ie, the representative pleasant odor) was presented via the olfactometer. Subjects were instructed to follow the auditory instructions for breathing and to breathe regularly without sniffing. ${ }^{19,20}$ Subjects were not trained to become familiar with the stimulation paradigm before the experiment; instead, before odor delivery at the start of each session, subjects were given 15 seconds to become familiar with the auditory respiration instructions. After the citral stimulation, $0.2 \mathrm{~mL}$ of $1 \mathrm{mmol} / \mathrm{L} \beta$-mercaptoethanol (BME) (ie, the representative unpleasant odor) was presented in the same fashion. There was a time interval of at least 30 minutes between 
the citral and BME sessions, to avoid possible habituation. The room was dark, with only dimmed light, with no other stimulation besides the odorants. After the fMRI examination, the subjects were asked to describe what the odor smelled like and to judge its pleasantness. All control subjects detected the odors, either citral or BME. All subjects with anosmia reported that they did not detect any odors during the examination.

Before undertaking this prospective study, to determine the odor concentration for the fMRI examination, we presented 2 heathy subjects with serial dilutions of citral or BME by the ascending method. ${ }^{21}$ Thus, detection and recognition thresholds were determined, and we set the recognition threshold as the stimulant concentration for our study.

\section{fMRI Data Analysis}

fMRI data preprocessing was performed with SPM8 software (http://www.fil.ion.ucl.ac.uk/spm/software/spm12). The first 4 volumes of each dataset were discarded to allow equilibration effects. Echo-planar images were corrected for slice-time differences and realigned to the first scan by rigid body transformation to correct for head movement. EPI and structural scans were normalized to the EPI standard template in the Montreal Neurological Institute (MNI) space (MNI: International Consortium for Brain Mapping) using linear and nonlinear transformations and were finally smoothed with a Gaussian kernel of $8-\mathrm{mm}$ full width at half maximum.

\section{Structural MR Imaging Analysis}

A neuroradiologist with 20 years of experience assessed the structural abnormality of the brain, blinded to the clinical data, using images from the T1-weighted fast-spoiled gradient-recalled sequence. For assessment, multiplanar reformatted images and original axial images were used. When tissue loss was present, the maximal size of the tissue loss was measured.

\section{Statistical Analysis}

To compare clinical variables between patients with anosmia and HC subjects, we used the nonparametric Mann-Whitney $U$ test in consideration of our small sample size. $P$ values $<.05$ were considered statistically significant. Data represent the mean $\pm \mathrm{SD}$ unless otherwise indicated. These statistical analyses were performed using the Statistical Package for the Social Sciences, Version 23.0. (SPSS; IBM, Armonk, New York).

Statistical analysis for fMRI was performed using general linear modeling implemented on SPM8 software. To provide a descriptive overview of the activation pattern (cluster size, $\geq 5$ voxels) of each group in each $\mathrm{fMRI}$ condition, we performed 1-sample $t$ tests with a false discovery rate-corrected threshold of $P<.05$. We then tested group differences using 2 -sample $t$ tests with age as a covariate in each stimulation condition, using the same statistical threshold. The voxels representing active structures were overlaid on 3D T1weighted anatomic images in MNI coordinates.

\section{RESULTS}

\section{Clinical Characteristics and Results of Olfactory Testing}

There was a significant difference in age between the anosmia group $(42.2 \pm 10.4$ years $)$ and the HC group $(29.3 \pm 8.5$ years $)$
$(P<.001)$; however, there was no between-group difference in sex ratio. The mean time between head injury and clinical consultation was 11.5 months (range, 10 days to 9 years) in the anosmia group. Four of 16 patients exhibited focal tissue loss on either side of the orbitofrontal cortex on structural MR imaging, with the size of the loss varying from $<1.0 \mathrm{~cm}$ in 3 patients to approximately $3.0 \mathrm{~cm}$ in 1 patient. In patients with anosmia, the mean CC-SIT score was $2.69 \pm 1.96$, the mean KVSS I score was $2.0 \pm 1.1$, and the mean KVSS II score was $3.2 \pm 2.9$, indicating that all patients met the diagnostic criteria for anosmia.

\section{fMRI Activation in the HC Group}

Citral stimulation produced activation in some olfaction-related structures, including the bilateral medial orbitofrontal gyri and left inferior frontal gyrus (On-line Figure). BME stimulation produced more robust activation in multiple olfactory structures, including the amygdala, piriform cortex, insula, and orbitofrontal cortex (Fig 2A). We also observed significant recruitment of the superior temporal gyrus, middle temporal gyrus, inferior parietal lobule, and precuneus.

\section{fMRI Activation in the Anosmia Group}

Citral stimulation produced activation in the orbitofrontal cortex and insula and multiple bilateral cortical association areas, including the prefrontal cortex, supramarginal gyrus, and superior temporal gyrus (On-line Figure). In contrast to the HC group, no significant clusters were identified in response to BME stimulation in the anosmia group (Fig 2B).

\section{Between-Group Comparison of fMRI Activation}

In the between-group comparison, there were more prominent differences in activation in response to BME stimulation compared with citral stimulation, with differences identified in multiple olfactory structures and associated cortices in the BME condition (On-line Table 1). In contrast, between-group differences in the citral condition were only observed in small clusters in the left inferior frontal gyrus and right superior frontal gyrus.

\section{Correlation between BOLD Activation and the KVSS-II Score}

A correlation analysis was performed on KVSS II scores and fMRI brain responses to both citral and BME stimulation in the anosmia group. In both conditions, a weak-but-significant positive correlation was identified between BOLD activation in the left insula and the KVSS-II score (uncorrected $P<.001$ ) (On-line Table 2).

\section{DISCUSSION}

The present study demonstrates that fMRI may have utility for detecting olfactory dysfunction after closed head trauma. Compared with HC subjects, patients with traumatic anosmia exhibited reduced BOLD activation in multiple olfactory structures and associated cortices. Furthermore, these changes were most clearly observed in response to the unpleasant odor stimulation with BME.

Since the introduction of olfactory fMRI by Yousem et al in 


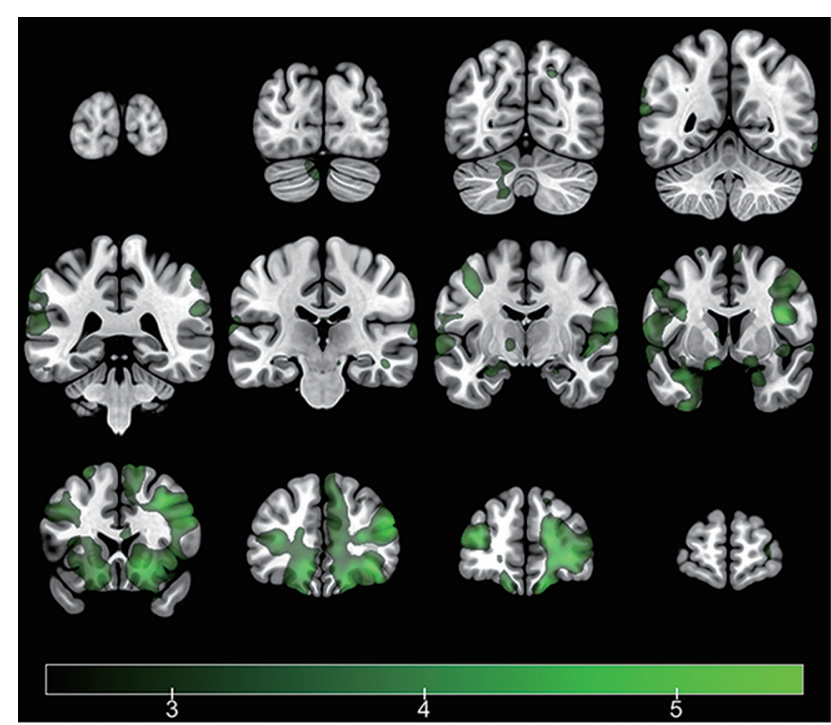

A

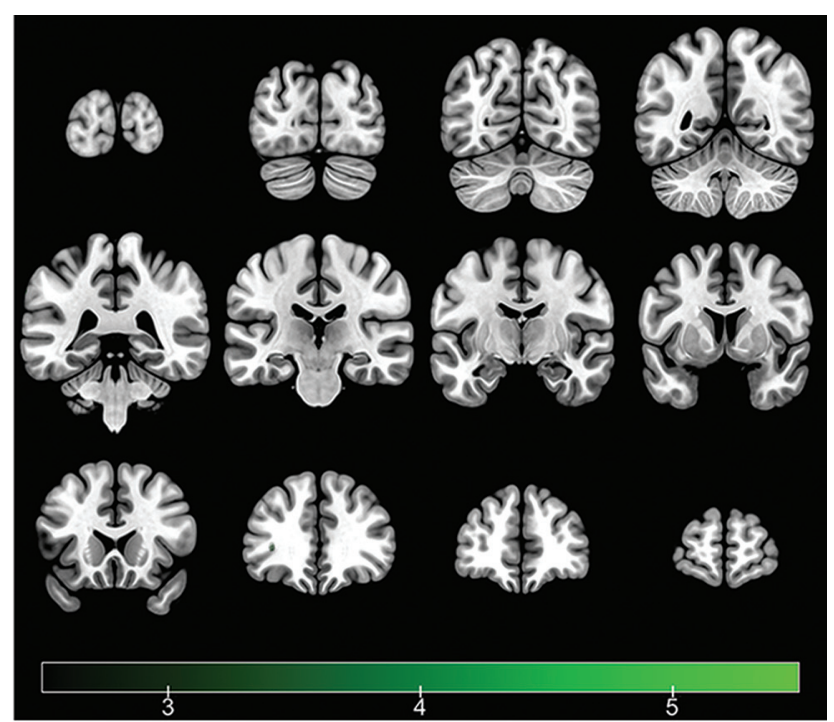

B

FIG 2. Representation of average brain-activation maps of the healthy control group $(A)$ and the traumatic anosmia group $(B)$ in response to the $\beta$-mercaptoethanol stimuli (1-sample $t$ test, voxelwise threshold false discovery rate-corrected $P<.05)(t$ values in a color scale). While the healthy control group shows activation in multiple olfactory structures and other associated brain regions, the traumatic anosmia group shows no activation in response to BME stimuli. The left side of the brain is presented on the left side of the image, according to neurologic convention.

$1997,{ }^{11}$ fMRI has become increasingly used as a reliable tool for assessing olfactory function and brain responses to olfactory stimulation in a number of disease states. ${ }^{15,16,22,23}$ In our study, we identified significant impairment in brain activation on the surface of the uncus housing the primary olfactory cortex ${ }^{24}$ and the orbitofrontal cortex as the secondary olfactory cortex as well as the insular cortex, which is associated with the processing of emotional aspects of odors, ${ }^{25}$ in patients with traumatic anosmia. A previous study similarly reported that unpleasant odors produced stronger activation in the left insula of right-handed subjects compared with pleasant odors, suggesting that insular activation is related to the subjective hedonic or aversive perception of odors. $^{26}$

Our finding of decreased brain activation in the bilateral temporal cortex and left superior parietal lobule of patients with traumatic anosmia in response to an unpleasant odor is consistent with previous observations. ${ }^{27-29}$ Although olfaction primarily functions as a means for odor perception and identification, it also serves an additional function in understanding and integrating multimodal actions. ${ }^{27}$ A previous fMRI study that used visual and olfactory stimuli reported increased activation in the middle temporal gyrus and parietal cortex (areas of multisensory integration) during stimulation, supporting the idea that olfaction serves multiple functions. ${ }^{28}$ Furthermore, activation in the temporoparietal cortex has also been associated with odor-recognition memory, a task with a high cognitive demand. ${ }^{30}$

The observation of a clear discrepancy in brain activation between the HC and anosmia groups in the unpleasant odor condition but not the pleasant odor condition can be explained by several different observations and hypotheses. First, our result is consistent with those of previous psychophysiological studies showing that unpleasant odors produce a greater degree of brain activation than pleasant odors. ${ }^{31,32}$ Second, previous studies have shown that the piriform cortex and other parts of the primary olfactory cortex are only briefly activated in response to odor stimulation ( $<10-15$ seconds), with BOLD signal decreasing to baseline shortly thereafter. ${ }^{27,33}$ Although our study design used a brief 10-second odor stimulus, a habituation effect would still be possible and may have obscured differences between the HC and anosmia groups in the pleasant odor condition (ie, in the condition evoking inherently weaker activation). Finally, while the unpleasant olfactory stimulation we used in this study is not known to cause trigeminal stimulation, clearly the pleasant odor of citral is related to trigeminal stimulation to a certain degree. ${ }^{34}$ Thus, mild activation of olfactory areas by citral may be related to intranasal trigeminal stimulation of citral instead of olfactory stimulation. This hypothesis is supported by our observation that citral stimulation in the anosmia group caused activation in multiple bilateral association areas, including the prefrontal cortex, supramarginal gyrus, and superior temporal gyrus, because these areas are involved in trigeminal stimulation. ${ }^{35}$

In contrast, the unpleasant BME invoked absolutely no activation in subjects with traumatic anosmia, thereby demonstrating an absence of trigeminal stimulation (if the trigeminal pathway could be stimulated at all in subjects with anosmia, we would have observed at least some activation in brain regions following BME applications). This result implies that the unpleasant BME odor may be a more suitable stimulant for detecting true subjects with anosmia because trigeminal stimulation can result in false-positive findings in the diagnosis of anosmia and should be minimized to decrease any activation not related to olfactory stimulation.

Finally, we observed a weak-but-significant positive correlation between activation in the left insular cortex and the KVSS II score in the pleasant and unpleasant stimulus conditions in the anosmia group. It is known that olfactory processes are lateralized in accordance with function in certain brain areas. Left hemispheric areas such as the orbitofrontal cortex, insula, piriform cortex, amygdala, and superior frontal cortex are primarily in-

AJNR Am J Neuroradiol 39:2320-25 Dec 2018 www.ajnr.org

2323 
volved with emotional responses, whereas right hemispheric areas are more involved with memory or familiarity ratings of olfaction. ${ }^{26,36}$ Therefore, left-sided activation of the insular cortex may be specific to the emotional processing or subjective perception of an unpleasant olfactory stimulus. Consistent with this hypothesis, a previous histopathologic study identified axonal projections from the primary and secondary olfactory cortical areas to the insula, ${ }^{37}$ and other fMRI studies have corroborated a relationship between olfaction and insular activation. ${ }^{38}$

The present study had several limitations. First, the statistical power of our results was rather limited by the small sample size. Therefore, future studies should confirm our findings in a larger cohort to determine the exact relationship between fMRI brain activation and clinical parameters in patients with traumatic anosmia. Second, the difference in age between groups might have contributed to reduced brain activation observed in the anosmia group because olfactory function is known to decline with increasing age. ${ }^{39}$ Thus, we treated age as a covariate in our analysis. Although age is reportedly related to olfactory function, age decline has not been noted until 60 years of age. ${ }^{20,39}$ In addition, some studies have argued that the ability to identify unpleasant odors is unrelated to age. ${ }^{31}$ Therefore, we believe that a between-group age difference did not affect our study results.

Although more expensive and less accessible than subjective psychophysical testing, fMRI has an important potential for objectively detecting alterations in brain activity related to olfaction. Of note, psychophysical test results can be compromised when patients with posttraumatic anosmia exhibit associated cognitive symptoms or malingering due to secondary gain, so fMRI may be more reliable in patients with traumatic anosmia. Olfactory event-related potentials have also been suggested as an objective assessment tool for identifying olfactory deficits ${ }^{36}$; however, this electrophysiologic approach lacks a methodology capable of producing a selective and controlled stimulation of the olfactory system. ${ }^{40}$ Olfactory bulb volumetry using high-resolution structural imaging ( $<0.5-\mathrm{mm}$ resolution) can be one of the objective measures of olfactory dysfunction. However, the normative value of olfactory bulb volume has not been established yet, and the assessment is mainly dependent on the subjective visual analysis by experts. ${ }^{41}$ In this regard, a further study of a larger sample group, including both ultra-high-resolution structural imaging for olfactory volumetry and olfactory fMRI, will be needed.

\section{CONCLUSIONS}

Our findings support the utility of olfactory fMRI for the objective visualization of deficits in olfaction-related brain activation in patients with traumatic anosmia. Future studies should confirm our preliminary findings regarding the diagnostic utility of olfactory fMRI.

Disclosures: Jin Kook Kim—RELATED: Grant: This research was supported by the Basic Science Research Program through the National Research Foundation of Korea funded by the Ministry of Education (NRF-2016RIDIA1B01012705)*. *Money paid to the institution.

\section{REFERENCES}

1. Costanzo RM, Miwa T. Posttraumatic olfactory loss. Adv Otorhinolaryngol 2006;63:99-107 Medline
2. Miao X, Yang L, Gu H, et al. Evaluation of post-traumatic anosmia with MRI and chemosensory ERPs. Eur Arch Otorhinolaryngol 2015; 272:1945-53 CrossRef Medline

3. Schofield PW, Moore TM, Gardner A. Traumatic brain injury and olfaction: a systematic review. Front Neurol 2014;5:5 CrossRef Medline

4. Murphy C, Morgan CD, Geisler MW, et al. Olfactory event-related potentials and aging: normative data. Int J Psychophysiol 2000;36: 133-45 CrossRef Medline

5. Bailie JM, Rybalsky KA, Griffith NM, et al. The susceptibility of olfactory measures to malingering. Chemosensory Perception 2008;1: 168-73 CrossRef

6. Yousem DM, Geckle RJ, Bilker WB, et al. Posttraumatic olfactory dysfunction: MR and clinical evaluation. AJNR Am J Neuroradiol 1996;17:1171-79 Medline

7. Sigurdardottir S, Jerstad T, Andelic N, et al. Olfactory dysfunction, gambling task performance and intracranial lesions after traumatic brain injury. Neuropsychology 2010;24:504-13 CrossRef Medline

8. Ciofalo A, De Vincentiis M, Iannella G, et al. Mild traumatic brain injury: evaluation of olfactory dysfunction and clinical-neurological characteristics. Brain Inj 2018;32:550-56 CrossRef Medline

9. Newton MR, Greenwood RJ, Britton KE, et al. A study comparing SPECT with CT and MRI after closed head injury. J Neurol Neurosurg Psychiatry 1992;55:92-94 CrossRef Medline

10. Atighechi S, Salari H, Baradarantar MH, et al. A comparative study of brain perfusion single-photon emission computed tomography and magnetic resonance imaging in patients with post-traumatic anosmia. Am J Rhinol Allergy 2009;23:409-12 CrossRef Medline

11. Yousem DM, Williams SC, Howard RO, et al. Functional MR imaging during odor stimulation: preliminary data. Radiology 1997;204: 833-38 CrossRef Medline

12. Levy LM, Henkin RI, Lin CS, et al. Odor memory induces brain activation as measured by functional MRI. J Comput Assist Tomogr 1999;23:487-98 CrossRef Medline

13. Schneider F, Habel U, Reske M, et al. Neural substrates of olfactory processing in schizophrenia patients and their healthy relatives. Psychiatry Res 2007;155:103-12 CrossRef Medline

14. Westermann B, Wattendorf E, Schwerdtfeger U, et al. Functional imaging of the cerebral olfactory system in patients with Parkinson's disease. J Neurol Neurosurg Psychiatry 2008;79:19-24 Medline

15. Wang J, Eslinger PJ, Doty RL, et al. Olfactory deficit detected by fMRI in early Alzheimer's disease. Brain Res 2010;1357:184-94 CrossRef Medline

16. Vasavada MM, Martinez B, Wang J, et al. Central olfactory dysfunction in Alzheimer's disease and mild cognitive impairment: a functional MRI study. J Alzheimers Dis 2017;59:359-68 CrossRef Medline

17. Cho JH, Jeong YS, Lee YJ, et al. The Korean version of the Sniffin' stick (KVSS) test and its validity in comparison with the cross-cultural smell identification test (CC-SIT). Auris Nasus Larynx 2009;36: 280-86 CrossRef Medline

18. Hong SC, Yoo YS, Kim ES, et al. Development of KVSS test (Korean version of Sniffin' sticks test). Korean J Otolaryngol-Head Neck Surg 1999;42:855-60

19. Sobel N, Prabhakaran V, Desmond JE, et al. Sniffing and smelling: separate subsystems in the human olfactory cortex. Nature 1998; 392:282-86 CrossRef Medline

20. Yousem DM, Maldjian JA, Hummel T, et al. The effect of age on odor-stimulated functional MR imaging. AJNR Am J Neuroradiol 1999;20:600-08 Medline

21. Doty RL, Smith R, McKeown DA, et al. Tests of human olfactory function: principal components analysis suggests that most measure a common source of variance. Percept Psychophys 1994;56: 701-07 CrossRef Medline

22. Henkin RI, Levy LM. Functional MRI of congenital hyposmia: brain activation to odors and imagination of odors and tastes. J Comput Assist Tomogr 2002;26:39-61 CrossRef Medline

23. Hummel T, Fliessbach K, Abele M, et al. Olfactory FMRI in patients 
with Parkinson's disease. Front Integr Neurosci 2010;4:125 CrossRef Medline

24. Zelano C, Bensafi M, Porter J, et al. Attentional modulation in human primary olfactory cortex. Nat Neurosci 2005;8:114-20 CrossRef Medline

25. Katata K, Sakai N, Doi K, et al. Functional MRI of regional brain responses to 'pleasant' and 'unpleasant' odors. Acta Otolaryngol Suppl 2009;85-90 Medline

26. Royet JP, Plailly J. Lateralization of olfactory processes. Chem Senses 2004;29:731-45 CrossRef Medline

27. Vedaei F, Fakhri M, Harirchian MH, et al. Methodological considerations in conducting an olfactory fMRI study. Behav Neurol 2013; 27:267-76 CrossRef Medline

28. Tubaldi F, Turella L, Pierno AC, et al. Smelling odors, understanding actions. Soc Neurosci 2011;6:31-47 CrossRef Medline

29. Savic I. Processing of odorous signals in humans. Brain Res Bull 2001;54:307-12 CrossRef Medline

30. Savic I, Gulyas B, Larsson M, et al. Olfactory functions are mediated by parallel and hierarchical processing. Neuron 2000;26:735-45 CrossRef Medline

31. Konstantinidis I, Hummel T, Larsson M. Identification of unpleasant odors is independent of age. Arch Clin Neuropsychol 2006;21: 615-21 CrossRef Medline

32. Alaoui-Ismaili O, Vernet-Maury E, Dittmar A, et al. Odor hedonics: connection with emotional response estimated by autonomic parameters. Chem Senses 1997;22:237-48 CrossRef Medline

33. Poellinger A, Thomas R, Lio $\mathrm{P}$, et al. Activation and habituation in olfaction: an fMRI study. Neuroimage 2001;13:547-60 Medline
34. Doty RL, Brugger WE, Jurs PC, et al. Intranasal trigeminal stimulation from odorous volatiles: psychometric responses from anosmic and normal humans. Physiol Behav 1978;20:175-85 CrossRef Medline

35. Albrecht J, Kopietz R, Frasnelli J, et al. The neuronal correlates of intranasal trigeminal function-an ALE meta-analysis of human functional brain imaging data. Brain Res Rev 2010;62:183-96 CrossRef Medline

36. Barresi M, Ciurleo R, Giacoppo S, et al. Evaluation of olfactory dysfunction in neurodegenerative diseases. J Neurol Sci 2012;323:16-24 CrossRef Medline

37. Jones-Gotman M, Zatorre RJ. Olfactory identification deficits in patients with focal cerebral excision. Neuropsychologia 1988;26:387400 CrossRef Medline

38. Fulbright RK, Skudlarski P, Lacadie CM, et al. Functional MR imaging of regional brain responses to pleasant and unpleasant odors. AJNR Am J Neuroradiol 1998;19:1721-26 Medline

39. Doty RL, Kamath V. The influences of age on olfaction: a review. Front Psychol 2014;5:20 CrossRef Medline

40. Rombaux P, Mouraux A, Bertrand B, et al. Assessment of olfactory and trigeminal function using chemosensory event-related potentials. Neurophysiol Clin 2006;36:53-62 CrossRef Medline

41. Chung MS, Choi WR, Jeong HY, et al. MR imaging-based evaluations of olfactory bulb atrophy in patients with olfactory dysfunction. AJNR Am J Neuroradiol 2017 Dec 21. [Epub ahead of print] CrossRef Medline 